\title{
Fault Detection for a Class of Impulsive Switched Systems
}

\author{
Qing-Yu Su Yan-Cheng Li Xing-Ze Dai Jian Li \\ School of Automation Engineering, Northeast Dianli University, Jilin 132012, China
}

\begin{abstract}
The problem of fault detection for a class of nonlinear impulsive switched systems is investigated in this paper. Fault detection filters are designed such that the augmented systems are stable, and the residual error signal generated by the filters guarantees the $H_{\infty}$ performance for disturbances and faults. Sufficient conditions for the design of fault detection (FD) filters are presented by linear matrix inequalities. Moreover, the filter gains are characterized according to a solution of a convex optimization. Finally, an example derived from a pulse-width-modulation-driven boost converter is given to illustrate the effectiveness of the FD design approach.
\end{abstract}

Keywords: Impulsive switched systems, fault detection (FD), $H_{\infty}$ performance, dwell time, filter design.

\section{Introduction}

Because of the higher demands for safety and reliability of control systems, fault detection (FD) is a significant problem in engineering ${ }^{[1,2]}$. Currently, there exist many results on this issue, such as using robust $H_{\infty}$ technique $^{[3]}$ and $H_{\infty} / H_{-}$technique $^{[4,5]}$

On the other hand, switched systems are the most typical class of hybrid systems ${ }^{[6-8]}$, mainly because many practical systems, such as aeronautics and astronautics systems ${ }^{[9]}$, networked control systems ${ }^{[10]}$, chemical systems ${ }^{[11]}$, can be modeled as switched systems. Many methods have been developed in the study of switched systems such as common Lyapunov function ${ }^{[12]}$, multiple Lyapunov functions ${ }^{[13]}$, and switched Lyapunov functions ${ }^{[14]}$. Furthermore, as an important class of switched systems, impulsive switched systems have received considerable attention. There appears some numbers of papers have focused on stability problem of nonlinear impulsive switched systems ${ }^{[15-17]}$.

Compared with the stability and the stabilization problems ${ }^{[18]}$, the FD design problem in switched systems is still in the early stage of development ${ }^{[19,20]}$, let alone the impulsive switched systems. Moreover, since the existing approaches about FD problem or switched systems are not appropriate for FD problem for nonlinear impulsive switched systems, the new technique should solve the impulsive case.

Motivated by the above reasons, in this paper, the problem of FD filters for a class of nonlinear impulsive switched systems is addressed in this paper. Different from [21], attention is focused on the design of FD filters guaranteeing a prescribed disturbance attenuation level in the $H_{\infty}$ performance. The main contributions of this paper include the following two points. First, the $H_{\infty}$ performance of this class of nonlinear impulsive switched systems with dwell time to reflect the effect of the residual error signal from disturbances and faults is proposed. Subsequently, the sufficient condition for the solvability of the FD filters is established by linear matrix inequalities (LMIs).

Manuscript received June 5, 2013; revised September 30, 2013

This work was supported by Northeast Dianli University (Nos. BSJXM09 and BSJXM10).
The paper is organized as follows. Section 2 introduces the problem under consideration and presents the design objectives. Section 3 illustrates the FD filter design approach in detail. An example is given in Section 4 to demonstrate the proposed method. Conclusions of this paper are given in the last section.

Notations: For a matrix $A, A^{\mathrm{T}}$ denotes its transpose. For a symmetric matrix, $A>0(A \geqslant 0)$ and $A<0$ $(A \leqslant 0)$ denote positive-definite (positive semi-definite) matrix and negative-definite (negative semi-definite) matrix, respectively. The Hermitian part of a square matrix $M$ is denoted by $H e(M)=M+M^{\mathrm{T}}$. The symbol $*$ within a matrix represents the symmetric entries.

\section{Problem statement}

\subsection{System description}

Consider the following impulsive switched systems:

$$
\left\{\begin{array}{rr}
\dot{x}(t)=\sum_{j=1}^{N} \xi_{j}(t)\left(A_{j} x(t)+B_{j 1} d(t)+B_{j 2} f(t)+\right. & \\
\left.\Gamma_{j}(t, x(t))\right), & t \neq t_{k} \\
\Delta x(t)=\sum_{j=1}^{N} \xi_{j}(t)\left(H_{j} x(t)+\Lambda_{j} x(t)(t, x(t))\right), & t=t_{k} \\
y(t)=\sum_{j=1}^{N} \xi_{j}(t)\left(C_{j} x(t)+D_{j 1} d(t)+D_{j 2} f(t)\right), & x\left(t_{0}^{+}\right)=x_{0}
\end{array}\right.
$$

where $x(t) \in \mathbf{R}^{n}$ is the state, $y(t) \in \mathbf{R}^{m}$ is the measured output, $d(t) \in \mathbf{R}^{n_{d}}$ and $f(t) \in \mathbf{R}^{n_{f}}$ are the disturbance input and the fault input, respectively. It is assumed that both the disturbance input and the fault input are energy bounded, then it demands that they belong to $l_{2}[0, \infty)$. The positive integer $N>1$ is the number of subsystems, and $j$ the switching signal which specifies which subsystem is activated at the switching instant. $\xi_{j}(t): Z^{+} \rightarrow\{0,1\}$ satisfies $\sum_{j=1}^{N} \xi_{j}(t)=1, t>0, j \in \mathbf{N}=\{1, \cdots, N\}$. The positive integers $k$ and $t_{k}$ denote the switching times and the switching instant for $k$-th switching, respectively. The 
matrices $A_{j}, B_{j 1}, B_{j 2}, C_{j}, D_{j 1}$ and $D_{j 2}$ have appropriate dimensions. $\Gamma_{j}(t, x(t)):\left[t_{0}, \infty\right) \times \mathbf{R}^{n} \rightarrow \mathbf{R}^{n}$, which is globally Lipschitz continuous, $\Gamma_{j}(t, 0)=0$ for all $t \in\left[t_{0}, \infty\right)$, and, $\Delta x\left(t_{k}\right)=x\left(t_{k}^{+}\right)-x\left(t_{k}^{-}\right)=x\left(t_{k}^{+}\right)-x\left(t_{k}\right)$, with $x\left(t_{k}^{+}\right)=$ $\lim _{t \rightarrow t_{k}^{+}} x(t)$ and $x\left(t_{k}\right)=x\left(t_{k}^{-}\right)=\lim _{t \rightarrow t_{k}^{-}} x(t)$, i.e., the solution $x(t)$ is left continuous. $\Lambda_{j}(t, x(t)):\left[t_{0}, \infty\right) \times \mathbf{R}^{n} \rightarrow$ $\mathbf{R}^{n}$ is a nonlinear function, and $\Lambda_{j}(t, 0) \equiv 0$ for all $t \in$ $\left[t_{0}, \infty\right)$.

Next, we will introduce the following assumptions for nonlinear impulsive switched systems (11).

Assumption 1. There exist some nonnegative scalars $g_{j}>0, j \in \mathbf{N}$, such that

$$
\Gamma_{j}^{\mathrm{T}}(t, x(t)) \Gamma_{j}(t, x(t)) \leqslant g_{j} x^{\mathrm{T}}(t) x(t) .
$$

Assumption 2. Denote $\rho_{j}(\cdot)$ as the spectral radius for each subsystem, then it holds that

$$
\left\|\Lambda_{j} x(t)(t, x(t))\right\| \leqslant \rho_{j}\left(H_{j}+1\right)\|x(t)\|, \quad t \in\left[t_{0}, \infty\right) .
$$

For the purpose of the fault detection, the following FD filters are designed

$$
\left\{\begin{array}{c}
\dot{x}_{f}(t)=\sum_{j=1}^{N} \xi_{j}(t)\left(A_{f j} x_{f}(t)+B_{f j} y(t)\right) \\
r(t)=\sum_{j=1}^{N} \xi_{j}(t)\left(C_{f j} x_{f}(t)+D_{f j} y(t)\right)
\end{array}\right.
$$

where $x_{f}(t)$ is the state of the filter, $r(t)$ is the residual signal. The matrices $A_{f j}, B_{f j}, C_{f j}$, and $D_{f j}$ with appropriate dimensions are to be determined.

Remark 1. The FD filters depend on the switched system (1). The switching signal is prior designed, then filters switch synchronously with the switched system (1).

For fault detection, it is not necessary to estimate the fault $f(t)$ directly. We can formulate the fault as the weighted fault $\hat{f}(t)=W_{f}(s) f(t)$ with a given stable weighted matrix $W_{f}(s)$. Supposed that the minimal realization of $W_{f}(s)$ is

$$
\begin{aligned}
\dot{\bar{x}}(t) & =A_{w j} \bar{x}(t)+B_{w j} f(t) \\
\hat{f}(t) & =C_{w j} \bar{x}(t)+D_{w j} f(t)
\end{aligned}
$$

where $\bar{x}(t)$ is the state of the weighted fault, and $A_{w j}, B_{w j}$, $C_{w j}, D_{w j}$ are the known constant matrices.

Remark 2. In practical applications, the frequency characteristics of faults is in a limited ranger. Then similar to $[20,21]$, the stable weighted matrix $W_{f}(s)$ is introduced to improve the system performance and emphasize the captured frequency characteristics of faults.

Denote $e(t)=r(t)-\hat{f}(t)$ and combine the states of the model of (1)-(3), then the augmented nonlinear impulsive switched system can be written as

$$
\begin{cases}\dot{\tilde{x}}(t)=\sum_{j=1}^{N} \xi_{j}(t)\left(\mathscr{A}_{j} \tilde{x}(t)+\mathscr{B}_{j} w(t)+\mathscr{T}_{j}(t, \tilde{x}(t))\right), & t \neq t_{k} \\ \Delta \tilde{x}(t)=\sum_{j=1}^{N} \xi_{j}(t)\left(\mathscr{H}_{j} \tilde{x}(t)+\mathscr{J}_{j}(t, \tilde{x}(t))\right), & t=t_{k} \\ e(t)=\sum_{j=1}^{N} \xi_{j}(t)\left(\mathscr{C}_{j} \tilde{x}(t)+\mathscr{D}_{j} w(t)\right) & \end{cases}
$$

where

$$
\begin{aligned}
\tilde{x}(t) & =\left[x^{\mathrm{T}}(t) x_{f}^{\mathrm{T}}(t), \bar{x}^{\mathrm{T}}(t)\right]^{\mathrm{T}}, \\
w(t) & =[d(t), f(t)], \\
\Delta \tilde{x}\left(t_{k}\right) & =\tilde{x}\left(t_{k}^{+}\right)-\tilde{x}\left(t_{k}^{-}\right)=\tilde{x}\left(t_{k}^{+}\right)-\tilde{x}\left(t_{k}\right), \\
\mathscr{H}_{j} & =\left[\begin{array}{ccc}
H_{j} & 0 & 0 \\
0 & 0 & 0 \\
0 & 0 & 0
\end{array}\right]
\end{aligned}
$$

and the equation at the bottom of this page.

Remark 3. Since $e(t)=r(t)-\hat{f}(t)$, residual signal $r(t)$ estimates fault $\hat{f}(t)$. By the stable weighting matrix $W_{f}(s)$, residual signal $r(t)$ estimates fault $f(t)$ to realize fault detection.

\subsection{Design objective}

The frameworks of FD filter design: the FD filters (2) are designed such that nonlinear impulsive switched system (4) is stable, residual errors between the residual signals and the faults are minimized for disturbances and faults. Our design objective of the FD filters can now be formulated as the following performances:

$$
\int_{t_{0}}^{\infty}\|e(s)\|^{2} \mathrm{~d} s \leqslant \gamma^{2} \int_{t_{0}}^{\infty}\|w(s)\|^{2} \mathrm{~d} s .
$$

Remark 4. Condition (5) is expressed to minimize the effects of the disturbance $d(t)$ and the fault $f(t)$ on residual errors $e(t)$ for impulsive switched system (4).

After designing the residual generator, it has to evaluate the residual evaluation function $J_{r}(t)$ and choose a threshold. Similarly to that proposed in [22], one of the widely adopted approaches is to select a residual evaluation function and a threshold:

1) The residual evaluation function is

$$
J_{r}(\tau)=\sqrt{\frac{1}{\tau} \sum_{s=s_{l}}^{s_{l}+\tau} r^{\mathrm{T}}(s) r(s)}
$$

where $J_{r}(\tau)$ is square value which means the average energy of residual signal over a time interval $\tau$, and $s_{l}$ denotes the initial evaluation time instant.

$$
\left[\begin{array}{c|c|c}
\mathscr{A}_{j} & \mathscr{B}_{j} & \mathscr{T}_{j}(t, \tilde{x}(t)) \\
\hline \mathscr{C}_{j} & \mathscr{D}_{j} & \mathscr{J}_{j}(t, \tilde{x}(t))
\end{array}\right]=\left[\begin{array}{ccc|cc|c}
A_{j} & 0 & 0 & B_{j 1} & B_{j 2} & \Gamma_{j}(t, x(t)) \\
B_{f j} C_{j} & A_{f j} & 0 & B_{f j} D_{j 1} & B_{f j} D_{j 2} & 0 \\
0 & 0 & A_{w j} & 0 & B_{w j} & 0 \\
\hline D_{f j} C_{j} & C_{f j} & -C_{w j} & D_{f j} D_{j 1} & D_{f j} D_{j 2}-D_{w j} & \Lambda_{j}(t, x(t))
\end{array}\right] .
$$


2) The threshold is

$$
J_{t h}=\sup _{\substack{d(t) \in l_{2}[0, \infty) \\ f(t)=0, j \in \mathbf{N}}} J_{r}
$$

Consequently, the occurrence of faults can be detected by the following logic rule.

$$
\left\{\begin{array}{l}
\left\|J_{r}(t)\right\| \leqslant J_{t h} \Rightarrow \text { no alarm } \\
\left\|J_{r}(t)\right\|>J_{t h} \Rightarrow \text { alarm. }
\end{array}\right.
$$

\section{The fault detection filter design}

Before beginning this section, the following lemmas are needed to present our main result.

Lemma $1^{[23]}$. Let $\epsilon>0$ be a given scalar and $\Xi \in \mathbf{R}^{p \times q}$ be a matrix such that $\Xi^{\mathrm{T}} \Xi \leqslant I$, where $I$ is an identity matrix with appropriate dimension. Then $2 x^{\mathrm{T}} \Xi y \leqslant$ $\epsilon x^{\mathrm{T}} x+\epsilon^{-1} y^{\mathrm{T}} y$ for all $x \in \mathbf{R}^{p}$ and $y \in \mathbf{R}^{q}$.

Lemma $\mathbf{2}^{[5]}$. Given a symmetric matrix $\Theta \in \mathbf{R}^{n \times n}$, and two matrices $\mathcal{M}$ and $\mathcal{H}$ of column dimension $n$, there exists matrix $\mathcal{F}$ that satisfies $\Theta+\mathcal{M}^{\mathrm{T}} \mathcal{F} \mathcal{H}+\mathcal{H}^{\mathrm{T}} \mathcal{F}^{\mathrm{T}} \mathcal{M}<0$ if and only if the following two conditions hold

$$
\mathcal{N}_{\mathcal{M}}^{\mathrm{T}} \Theta \mathcal{N}_{\mathcal{M}}<0, \quad \mathcal{N}_{\mathcal{H}} \Theta \mathcal{N}_{\mathcal{H}}^{\mathrm{T}}<0
$$

where $\mathcal{N}_{\mathcal{M}}$ and $\mathcal{N}_{\mathcal{H}}$ denote arbitrarily bases of null space of $\mathcal{M}$ and $\mathcal{H}$, respectively.

In this section, $H_{\infty}$ performance for nonlinear impulsive switched system (4) is given, and a sufficient condition for the solvability of the FD filters is established, and the desired filters can be obtained by solving a convex optimization problem.

Lemma 3. Let $\alpha_{j}, \varepsilon$ be constants satisfying $0<\alpha_{j}<$ $1, \varepsilon>0, j \in \mathbf{N}$ and Assumptions $1-2$ hold. Furthermore, suppose that nonlinear impulsive switched system (4) switches from $i$-th subsystem to $j$-th subsystem at switched time point $t_{k}$. If there exist Lyapunov functions candidate $V_{j}(t)=\tilde{x}(t)^{\mathrm{T}} \mathscr{P}_{j} \tilde{x}(t), \quad j \in \mathbf{N}$ satisfying the following inequalities (12), then nonlinear impulsive switched system (4) is stable with $H_{\infty}$-gain $\gamma$ for any switching signal satisfying

$$
\ln \left(\left.\mu_{i j}\right|_{k}\right)-\alpha_{i}\left(t_{k}-t_{k-1}\right)<0
$$

where $i \in \mathbf{N}, j \in \mathbf{N}, i \neq j, k=1,2, \cdots$, and $\left.\mu_{i j}\right|_{k}=$ $\frac{(\delta+1) \lambda_{\max }\left\{\left(\mathscr{H}_{j}+I\right)^{\mathrm{T}} \mathscr{P}_{j}\left(\mathscr{H}_{j}+I\right)\right\}+\left(\delta^{-1}+1\right) \rho_{j}^{2} \lambda_{\max }\left\{\mathscr{P}_{j}\right\}}{\lambda_{\min }\left\{\mathscr{P}_{i}\right\}}, \delta>0$.

Proof. When $t \in\left(t_{k}, t_{k+1}\right]$ and $j$-th subsystem is activated, evaluating the time derivative of $V_{j}(t)$ along the trajectory of nonlinear impulsive switched system (4) gives

$$
\begin{aligned}
\dot{V}_{j}(t)= & \tilde{x}^{\mathrm{T}}(t)\left(\mathscr{A}_{j}^{\mathrm{T}} \mathscr{P}_{j}+\mathscr{P}_{j} \mathscr{A}_{j}\right) \tilde{x}(t)+\tilde{x}^{\mathrm{T}}(t) \mathscr{P}_{j} \mathscr{B}_{j} w(t)+ \\
& w(t)^{\mathrm{T}} \mathscr{B}_{j}^{\mathrm{T}} \mathscr{P}_{j} \tilde{x}(t)+\mathrm{T}_{j}(t, \tilde{x}(t))^{\mathrm{T}} \mathscr{P}_{j} \tilde{x}(t)+ \\
& \tilde{x}^{\mathrm{T}}(t) \mathscr{P}_{j}(t, \tilde{x}(t)) .
\end{aligned}
$$

By Lemma 1, it is clear that

$$
\begin{aligned}
2 \mathscr{T}_{j}(t, \tilde{x}(t))^{\mathrm{T}} \mathscr{P}_{j} \tilde{x}(t) \leqslant \varepsilon \mathscr{T}_{j}(t, \tilde{x}(t))^{\mathrm{T}} \mathscr{T}_{j}(t, \tilde{x}(t))+ \\
\varepsilon^{-1} \tilde{x}^{\mathrm{T}}(t) \mathscr{P}_{j}^{2} \tilde{x}(t) .
\end{aligned}
$$

Therefore, when assuming the zero input (i.e., $w(t)=0$ ) and using Assumption 1, we have the following condition from (7) and (8),

$$
\dot{V}_{j} \|_{w(t)=0}(t) \leqslant \tilde{x}^{\mathrm{T}}(t) \mathcal{Q}_{j} \tilde{x}(t)
$$

where $\mathcal{Q}_{j}=\mathscr{A}_{j}^{\mathrm{T}} \mathscr{P}_{j}+\mathscr{P}_{j} \mathscr{A}_{j}+\varepsilon g_{j} I+\varepsilon^{-1} \mathscr{P}_{j}^{2}$. It follows from (12) that $\mathcal{Q}_{j} \leqslant-\alpha_{j} \mathscr{P}_{j} \leqslant 0$. Thus, from (9), we have $\left.\dot{V}_{j}(t)\right|_{w(t)=0}(t) \leqslant-\alpha_{j} V_{j}(t) \leqslant 0$. Integrating this inequality gives

$$
V_{j}(t) \leqslant \mathrm{e}^{-\alpha_{j}\left(t-t_{k}\right)} V_{j}\left(t_{k}^{+}\right), \quad t \in\left(t_{k}, t_{k+1}\right] .
$$

At the impulsive switching time point $t_{k}$, it has

$$
\begin{aligned}
V_{j}\left(t_{k}^{+}\right)= & \left(\Delta \tilde{x}\left(t_{k}\right)+\tilde{x}\left(t_{k}\right)\right)^{\mathrm{T}} \mathscr{P}_{j}\left(\Delta \tilde{x}\left(t_{k}\right)+\tilde{x}\left(t_{k}\right)\right)= \\
& {\left[\left(\mathscr{H}_{j}+I\right) \tilde{x}\left(t_{k}\right)\right]^{\mathrm{T}} \mathscr{P}_{j}\left[\left(\mathscr{H}_{j}+I\right) \tilde{x}\left(t_{k}\right)\right]+} \\
& 2 \mathscr{J}_{j}^{\mathrm{T}}(t, \tilde{x}(t)) \mathscr{P}_{j}\left[\left(\mathscr{H}_{j}+I\right) \tilde{x}\left(t_{k}\right)\right]+ \\
& \mathscr{J}_{j}^{\mathrm{T}}(t, \tilde{x}(t)) \mathscr{J}_{j}(t, \tilde{x}(t)) .
\end{aligned}
$$

By Lemma 2 in [24], it has

$$
\begin{aligned}
V_{j}\left(t_{k}^{+}\right) \leqslant & (\delta+1)\left[\left(\mathscr{H}_{j}+I\right) \tilde{x}\left(t_{k}\right)\right]^{\mathrm{T}} \mathscr{P}_{j}\left(\mathscr{H}_{j}+I\right) \tilde{x}\left(t_{k}\right)+ \\
& \left(\delta^{-1}+1\right) \mathscr{J}_{j}^{\mathrm{T}}(t, \tilde{x}(t)) \mathscr{P}_{j} \mathscr{J}_{j}(t, \tilde{x}(t)) \leqslant \\
& \left\{(\delta+1) \lambda_{\max }\left\{\left(\mathscr{H}_{j}+I\right)^{\mathrm{T}} \mathscr{P}_{j}\left(\mathscr{H}_{j}+I\right)\right\}+\right. \\
& \left.\left(\delta^{-1}+1\right) \rho_{j}^{2} \lambda_{\max }\left\{\mathscr{P}_{j}\right\}\right\} \tilde{x}^{\mathrm{T}}\left(t_{k}\right) \tilde{x}\left(t_{k}\right) \leqslant \\
& \left.\mu_{i j}\right|_{k} V_{i}\left(t_{k}\right) .
\end{aligned}
$$

Therefore, from (10) and (11), we have

$$
\begin{aligned}
V_{j}(t) \leqslant & \mathrm{e}^{-\alpha_{j}\left(t-t_{k}\right)} V_{j}\left(t_{k}^{+}\right) \leqslant \\
& \left.\mu_{i j}\right|_{k} \mathrm{e}^{-\alpha_{j}\left(t-t_{k}\right)} V_{i}\left(t_{k}\right) \leqslant \cdots \leqslant \\
& \left(\left.\prod_{\substack{l=1, q \neq p \\
q \in \mathbf{N}, p \in \mathbf{N}}}^{k} \mu_{q p}\right|_{l} \mathrm{e}^{-\alpha_{q}\left(t_{l}-t_{l-1}\right)}\right) \mathrm{e}^{-\alpha_{j}\left(t-t_{k}\right)} V_{o}\left(t_{0}\right)
\end{aligned}
$$

where $V_{o}\left(t_{0}\right)$ means that the $o$-th subsystem is activated at the instant $t_{0},\left.\mu_{q p}\right|_{l}$ denotes the $l$-th switching impulsive value from $q$-th subsystem to $p$-th subsystem, $\alpha_{q}$ denotes the decay rate of Lyapunov function for $q$-th subsystem. Since (6) holds, i.e., there exists $\left.\mu_{q p}\right|_{l}>0$ such that $\ln \left(\left.\mu_{q p}\right|_{l}\right)-\alpha_{q}\left(t_{l}-t_{l-1}\right)<0$, then

$$
g\left(t-t_{0}\right)=-\alpha_{j}\left(t-t_{k}\right)+\sum_{\substack{l=1, q \neq p \\ q \in \mathbf{N}, p \in \mathbf{N}}}^{k} \ln \left(\mu_{q p} \mid l\right)-\alpha_{q}\left(t_{l}-t_{l-1}\right)<0 .
$$

$$
\left[\begin{array}{cc}
\mathscr{A}_{j}^{\mathrm{T}} \mathscr{P}_{j}+\mathscr{P}_{j} \mathscr{A}_{j}+\varepsilon^{-1} \mathscr{P}_{j}^{2}+\alpha_{j} \mathscr{P}_{j}+\varepsilon g_{j} I & \mathscr{P}_{j} \mathscr{B}_{j} \\
\mathscr{B}_{j}^{\mathrm{T}} \mathscr{P}_{j} & 0
\end{array}\right]+\left[\begin{array}{cc}
\mathscr{C}_{j}^{\mathrm{T}} \mathscr{C}_{j} & \mathscr{C}_{j}^{\mathrm{T}} \mathscr{D}_{j} \\
\mathscr{D}_{j}^{\mathrm{T}} \mathscr{C}_{j} & -\gamma^{2} I+\mathscr{D}_{j}^{\mathrm{T}} \mathscr{D}_{j}
\end{array}\right]<0, \quad j \in \mathbf{N}
$$


It follows that

$$
V_{j}(t) \leqslant \mathrm{e}^{g\left(t-t_{0}\right)} V_{o}\left(t_{0}\right) .
$$

Therefore, we conclude that $V_{j}(t)$ converges to zero as $t \rightarrow \infty$, then the nonlinear impulsive switched system (4) with $w(t)=0$ is stable.

Secondly, we establish the $H_{\infty}$ performance for nonlinear impulsive switched system (4). Consider the following performance index: $J=\int_{t_{0}}^{\infty}\left(\|e(s)\|^{2}-\gamma^{2}\|w(s)\|^{2}\right) \mathrm{d} s$.

For any nonzero $w(t) \in l_{2}\left[t_{0}, \infty\right)$ and zero initial condition $\tilde{x}\left(t_{0}\right)$, let $\Upsilon(t)=e^{\mathrm{T}}(t) e(t)-\gamma^{2} w^{\mathrm{T}}(t) w(t)$, and consider the Lyapunov functions as $V_{j}(t)=\tilde{x}(t)^{\mathrm{T}} \mathscr{P}_{j} \tilde{x}(t)$. For $j$-th subsystem, it has

$$
V_{j}(\tilde{x}(t))+\alpha_{j} V_{j}(\tilde{x}(t))+\Upsilon(t) \leqslant \mathcal{X}^{\mathrm{T}} \mathfrak{R}_{j} \mathcal{X}, j \in \mathbf{N},
$$

where

$$
\begin{aligned}
& \mathcal{X}=\left[\begin{array}{cc}
\tilde{x}(t)^{\mathrm{T}} & w(t)^{\mathrm{T}}
\end{array}\right]^{\mathrm{T}} \\
& \mathfrak{R}_{j}=\left[\begin{array}{cc}
\Xi_{j} & \mathscr{P}_{j} \mathscr{B}_{j}+\mathscr{C}_{j}^{\mathrm{T}} \mathscr{D}_{j} \\
\mathscr{B}_{j}^{\mathrm{T}} \mathscr{P}_{j}+\mathscr{D}_{j}^{\mathrm{T}} \mathscr{C}_{j} & -\gamma^{2} I+\mathscr{D}_{j}^{\mathrm{T}} \mathscr{D}_{j}
\end{array}\right] \\
& \Xi_{j}=\mathscr{A}_{j}^{\mathrm{T}} \mathscr{P}_{j}+\mathscr{P}_{j} \mathscr{A}_{j}+\varepsilon^{-1} \mathscr{P}_{j}^{2}+\alpha_{j} \mathscr{P}_{j}+\varepsilon g_{j} I+\mathscr{C}_{j}^{\mathrm{T}} \mathscr{C}_{j}
\end{aligned}
$$

and (12) is equivalent to $\mathfrak{R}_{j}<0$. Then, it has $V_{j}(\tilde{x}(t))+$ $\alpha_{j} V_{j}(\tilde{x}(t))+\Upsilon(t) \leqslant 0$. By iteration operation on the above inequality for $t \in\left(t_{k}, t_{k+1}\right]$, we have

$$
V_{j}(t) \leqslant \mathrm{e}^{-\alpha_{j}\left(t-t_{k}\right)} V_{j}\left(t_{k}^{+}\right)-\int_{t_{k}}^{t} \Upsilon(s) \mathrm{d} s .
$$

Along the same lines as the impulsive switching time point $t_{k}$, it has from (11) and (13) that

$$
\begin{aligned}
V_{j}(t) \leqslant & \mathrm{e}^{-\alpha_{j}\left(t-t_{k}\right)} V_{j}\left(t_{k}^{+}\right)-\int_{t_{k}}^{t} \Upsilon(s) \mathrm{d} s \leqslant \\
& \left.\mu_{i j}\right|_{k} \mathrm{e}^{-\alpha_{j}\left(t-t_{k}\right)} V_{i}\left(t_{k}\right)-\int_{t_{k}}^{t} \Upsilon(s) \mathrm{d} s \leqslant \cdots \leqslant \\
& \mathrm{e}^{g\left(t-t_{0}\right)} V_{o}\left(t_{0}\right)-\int_{t_{0}}^{t} \mathrm{e}^{g(t-s)} \Upsilon(s) \mathrm{d} s
\end{aligned}
$$

Under the zero initial condition, inequality (14) implies $\int_{t_{0}}^{t} \mathrm{e}^{g(t-s)} \Upsilon(s) \mathrm{d} s<0$. Due to $0<g(t-s)<1, s \in\left[t_{0}, t\right)$, it has $\int_{t_{0}}^{t} \Upsilon(s) \mathrm{d} s<\int_{t_{0}}^{t} \mathrm{e}^{g(t-s)} \Upsilon(s) \mathrm{d} s<0$, that is,

$$
\int_{t_{0}}^{t} e^{\mathrm{T}}(s) e(s) \mathrm{d} s \leqslant \gamma^{2} \int_{t_{0}}^{t} w^{\mathrm{T}}(s) w(s) \mathrm{d} s
$$

When $t \rightarrow \infty$, nonlinear impulsive switched system (4) has $H_{\infty}$ performance, which completes the proof.

Based on Lemma 1, the following theorem is given to obtain a sufficient condition in terms of linear matrix inequalities.

Theorem 1. Let $\alpha_{j}, \gamma$ and $\varepsilon$ be constants satisfying $0<\alpha_{j}<1, \gamma>0, \varepsilon>0$ and Assumptions 1-2 hold. If there exist matrix variables $X_{j}, Y_{j}, Z_{j}, R_{j}, K_{j}, L_{j}, M_{j}$, $S_{j}, T_{j}, E_{j}, F_{j}, G_{j}, N_{j}, \hat{A}_{f j}, \hat{B}_{f j}, \hat{C}_{f j}, \hat{D}_{f j}$ and symmetric positive-definite matrices

$$
\mathscr{P}_{j}=\left[\begin{array}{ccc}
P_{j 1} & P_{j 2} & P_{j 3} \\
* & P_{j 4} & P_{j 5} \\
* & * & P_{j 6}
\end{array}\right]>0, j \in \mathbf{N}
$$

satisfying the following inequalities

$$
\left[\begin{array}{ccccc}
\Theta_{1} & \Theta_{2} & \Theta_{3} & 0 & 0 \\
* & \Theta_{4} & \Theta_{5} & \mathscr{P}_{j} & \Theta_{6} \\
* & * & -\gamma^{2} I & 0 & D_{j 1}^{\mathrm{T}} \hat{D}_{f j}^{\mathrm{T}} \\
* & * & * & -\varepsilon^{-1} I & 0 \\
* & * & * & * & -I
\end{array}\right]<0, j \in \mathbf{N}
$$

where (see $\Theta_{2}, \Theta_{4}, \Theta_{6}$ at the bottom of this paper).

$$
\begin{aligned}
\Theta_{1} & =\left[\begin{array}{ccc}
-H e\left(X_{j}\right) & -Y_{j}-N_{j}^{\mathrm{T}} & -Z_{j}-R_{j}^{\mathrm{T}} \\
* & -H e\left(N_{j}\right) & -K_{j}^{\mathrm{T}} \\
* & * & -H e\left(L_{j}\right)
\end{array}\right], \\
\Theta_{3} & =\left[\begin{array}{cc}
X_{j}^{\mathrm{T}} B_{j 1}+\hat{B}_{f j} D_{j 1} & X_{j}^{\mathrm{T}} B_{j 2}+\hat{B}_{f j} D_{j 2}+R_{j}^{\mathrm{T}} B_{w j} \\
Y_{j}^{\mathrm{T}} B_{j 1}+\hat{B}_{f j} D_{j 1} & Y_{j}^{\mathrm{T}} B_{j 2}+\hat{B}_{f j} D_{j 2}+K_{j}^{\mathrm{T}} B_{w j} \\
Z_{j}^{\mathrm{T}} B_{j 1} & Z_{j}^{\mathrm{T}} B_{j 2}+L^{\mathrm{T}} B_{w j}
\end{array}\right], \\
\Theta_{5} & =\left[\begin{array}{cc}
M_{j}^{\mathrm{T}} B_{j 1}+\hat{B}_{f j} D_{j 1} & M_{j}^{\mathrm{T}} B_{j 2}+\hat{B}_{f j} D_{j 2}+E_{j}^{\mathrm{T}} B_{w j} \\
S_{j}^{\mathrm{T}} B_{j 1}+\hat{B}_{f j} D_{j 1} & S_{j}^{\mathrm{T}} B_{j 2}+\hat{B}_{f j} D_{j 2}+F_{j}^{\mathrm{T}} B_{w j} \\
T_{j}^{\mathrm{T}} B_{j 1} & T_{j}^{\mathrm{T}} B_{j 2}+G^{\mathrm{T}} B_{w j}
\end{array}\right],
\end{aligned}
$$

then for any switching signal satisfying (6), nonlinear impulsive switched system (4) is stable, and guarantees the $H_{\infty}$ performance (5). Moveover, if (15) is feasible, then the FD filter gains in form of (2) can be given by

$$
\left[\begin{array}{cc}
A_{f j} & B_{f j} \\
C_{f j} & D_{f j}
\end{array}\right]=\left[\begin{array}{cc}
N_{j} & 0 \\
0 & I
\end{array}\right]^{-\mathrm{T}}\left[\begin{array}{cc}
\hat{A}_{f j} & \hat{B}_{f j} \\
\hat{C}_{f j} & \hat{D}_{f j}
\end{array}\right]
$$

$$
\begin{gathered}
\Theta_{2}=\left[\begin{array}{ccc}
P_{j 1}-M_{j}+X_{j}^{\mathrm{T}} A_{j}+\hat{B}_{f j} C_{j} & P_{j 2}-S_{j}+\hat{A}_{f j} & P_{j 3}-T_{j}+R^{\mathrm{T}} \hat{A}_{w j} \\
P_{j 2}^{\mathrm{T}}-N_{j}+Y_{j}^{\mathrm{T}} A_{j}+\hat{B}_{f j} C_{j} & P_{j 4}-N_{j}+\hat{A}_{f j} & P_{j 5}+K^{\mathrm{T}} \hat{A}_{w j} \\
P_{j 3}^{\mathrm{T}}-E_{j}+Z_{j}^{\mathrm{T}} A_{j} & P_{j 5}^{\mathrm{T}}-F_{j} & P_{j 6}-G_{j}+L^{\mathrm{T}} \hat{A}_{w j}
\end{array}\right], \quad \Theta_{6}=\left[\begin{array}{c}
C_{j}^{\mathrm{T}} \hat{D}_{f j}^{\mathrm{T}} \\
\hat{C}_{f j}^{\mathrm{T}} \\
-C_{w j}^{\mathrm{T}}
\end{array}\right], \\
\Theta_{4}=\left[\begin{array}{ccc}
\alpha_{j} P_{j 1}+H e\left(M_{j}^{\mathrm{T}} A_{j}+\hat{B}_{f j} C_{j}\right)+\varepsilon g_{j} I & \alpha_{j} P_{j 2}+\hat{A}_{f j}+A_{j}^{\mathrm{T}} S_{j}+C_{j}^{\mathrm{T}} \hat{B}_{f j}^{\mathrm{T}} & \alpha_{j} P_{j 3}+E_{j}^{\mathrm{T}} A_{w j}+A_{j}^{\mathrm{T}} T_{j} \\
* & \alpha_{j} P_{j 4}+\varepsilon g_{j} I+H e\left(\hat{A}_{f j}\right) & \alpha_{j} P_{j 5}+F_{j}^{\mathrm{T}} A_{w j} \\
* & * & \alpha_{j} P_{j 5}+\varepsilon g_{j} I+H e\left(\hat{A}_{w j}^{\mathrm{T}} G_{j}\right)
\end{array}\right],
\end{gathered}
$$




\section{Proof. Denote}

$$
\mathscr{M}_{j}=\left[\begin{array}{ccc}
0 & \mathscr{P}_{j} & 0 \\
\mathscr{P}_{j} & \Upsilon_{j} & \mathscr{C}_{j}^{\mathrm{T}} \mathscr{D}_{j} \\
0 & \mathscr{D}_{j}^{\mathrm{T}} \mathscr{C}_{j} & -\gamma^{2} I+\mathscr{D}_{j}^{\mathrm{T}} \mathscr{D}_{j}
\end{array}\right]
$$

where $\Upsilon_{j}=\alpha \mathscr{P}_{j}+\varepsilon^{-1} \mathscr{P}_{j}^{2}+\varepsilon g_{j} I+\mathscr{C}_{j}^{\mathrm{T}} \mathscr{C}_{j}$

After some simple calculations, (12) can be rewritten as

$$
\left[\begin{array}{cc}
\mathscr{A}_{j} & \mathscr{B}_{j} \\
I & 0 \\
0 & I
\end{array}\right]^{\mathrm{T}} \mathscr{M}_{j}\left[\begin{array}{cc}
\mathscr{A}_{j} & \mathscr{B}_{j} \\
I & 0 \\
0 & I
\end{array}\right]<0
$$

On the other hand,

$$
\left[\begin{array}{l}
0 \\
0 \\
I
\end{array}\right]^{\mathrm{T}} \mathscr{M}_{j}\left[\begin{array}{l}
0 \\
0 \\
I
\end{array}\right]=-\gamma^{2} I+\mathscr{D}_{j}^{\mathrm{T}} \mathscr{D}_{j}<0
$$

Based on Lemma 2, it follows from (16) and (17) that

$$
\mathscr{M}_{j}+H e\left(\left[\begin{array}{c}
-I \\
\tilde{A}_{j}^{\mathrm{T}} \\
\tilde{B}_{j 1}^{\mathrm{T}}
\end{array}\right] \mathscr{W}_{j}\left[\begin{array}{ccc}
I & 0 & 0 \\
0 & I & 0
\end{array}\right]\right)<0
$$

where $\mathscr{W}_{j}$ introduced by Projection Lemma is the matrix variable of appropriate dimensions. Partition $\mathscr{W}_{j}$ as $\mathscr{W}_{j}=\left[\begin{array}{cc}\mathscr{W}_{j 1} & \mathscr{W}_{j 2}\end{array}\right]$, and $\mathscr{W}_{j 1}, \mathscr{W}_{j 2}$, respectively, as

$$
\mathscr{W}_{j 1}=\left[\begin{array}{ccc}
X_{j} & Y_{j} & 0 \\
N_{j} & N_{j} & 0 \\
R_{j} & K_{j} & L_{j}
\end{array}\right], \mathscr{W}_{j 2}=\left[\begin{array}{ccc}
M_{j} & S_{j} & T_{j} \\
N_{j} & N_{j} & 0 \\
E_{j} & F_{j} & G_{j}
\end{array}\right]
$$

Define $\hat{A}_{f j}=N_{j}^{\mathrm{T}} A_{f j}, \hat{B}_{f j}=N_{j}^{\mathrm{T}} B_{f j}$, and by Schur complement, (18) becomes (15). Hence if the conditions (15) hold, nonlinear impulsive switched system (4) is stable, and guarantees the $H_{\infty}$ performance (5), which completes the proof.

Remark 5. The $H_{\infty}$ performance has been transformed into some linear matrix inequality (LMI) conditions. The filter gains are characterized according to a solution of the feasible matrix variables.

\section{Example}

In this section, we present an example derived from a pulse-width-modulation (PWM)-driven boost converter, shown in Fig. 1, to illustrate the effectiveness of FD design approach. $e_{s}(t)$ is the source voltage, $L$ is the inductance, $C$ is the capacitance, and $R$ is the load resistance. The switch $s(t)$ is controlled by a PWM device and can switch at most once in each period $T$. As a typical circuit system, the converter is used to transform the source voltage into a higher voltage.

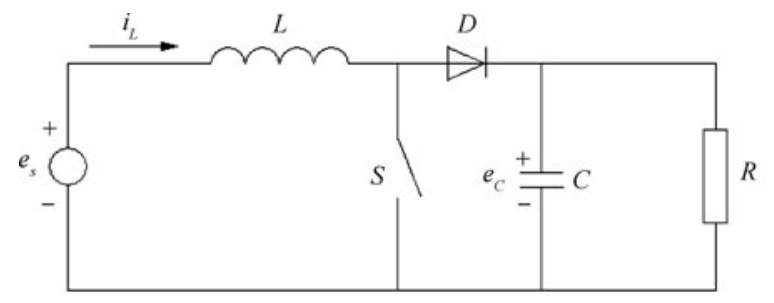

Fig. 1 Boost convertor

In recent years, this class of power converters also has been modeled as switched systems. The differential equations for the boost converter are as follows ${ }^{[25]}$ :

$$
\begin{aligned}
& \dot{e}_{C}(\tau)=-\frac{1}{R C} e_{C}(\tau)+(1-s(\tau)) \frac{1}{C_{1}} i_{L}(\tau), \\
& \dot{i}_{L}(\tau)=-(1-s(\tau)) \frac{1}{L_{1}} e_{C}(\tau)+s(\tau) \frac{1}{L_{1}} e_{s}(\tau)
\end{aligned}
$$

where $\tau=t / T, L_{1}=L / T$ and $C_{1}=C / T$.

Then, let $x=\left[e_{C}, i_{L}\right]^{\mathrm{T}}$, therefore, (19) and (20) can be formulated as

$$
\dot{x}(t)=\sum_{j=1}^{N} \xi_{j}(t)\left(A_{j} x(t)+B_{j} u(t)\right), \quad j \in\{1,2\}
$$

where

$$
\begin{aligned}
& A_{1}=\left[\begin{array}{cc}
-\frac{1}{R C_{1}} & \frac{1}{C_{1}} \\
-\frac{1}{L_{1}} & 0
\end{array}\right], A_{2}=\left[\begin{array}{cc}
-\frac{1}{R C_{1}} & 0 \\
0 & 0
\end{array}\right], \\
& B_{1}=\left[\begin{array}{l}
0 \\
0
\end{array}\right], B_{2}=\left[\begin{array}{c}
0 \\
\frac{1}{L_{1}}
\end{array}\right] .
\end{aligned}
$$

According to the same normalization technique used in [26], the closed-loop systems matrices can be given by

$$
A_{1}=\left[\begin{array}{ll}
-1 & 1 \\
-1 & 0
\end{array}\right], A_{2}=\left[\begin{array}{cc}
-1.0000 & 0 \\
0.5246 & -0.9779
\end{array}\right]
$$

We assume the matrices of the other systems to be

$$
\begin{aligned}
& B_{11}=\left[\begin{array}{c}
0.24 \\
-0.10
\end{array}\right], B_{12}=\left[\begin{array}{c}
0.95 \\
-1.29
\end{array}\right], \\
& B_{21}=\left[\begin{array}{c}
0.1 \\
-0.31
\end{array}\right], B_{22}=\left[\begin{array}{c}
-0.52 \\
1.34
\end{array}\right], \\
& \Gamma_{1}(t, x(t))=\left[\begin{array}{c}
\sin \left(x_{1}(t)\right) \mathrm{e}^{-0.05 t} \\
0
\end{array}\right], \\
& \Gamma_{2}(t, x(t))=\left[\begin{array}{c}
\sin \left(x_{1}(t)\right) \mathrm{e}^{-0.05 t} \\
\sin \left(x_{2}(t)\right) \mathrm{e}^{-0.05 t}
\end{array}\right], \\
& H_{1}=H_{2}=-\left[\begin{array}{c}
0.58 \quad 0 \\
0 \quad 0.58
\end{array}\right], \\
& \Lambda_{1}(t, x(t))=\Lambda_{2}(t, x(t))=\left[\begin{array}{c}
0.3 \sin \left(x_{1}\left(t_{i}\right)\right) \\
0.3 \sin \left(x_{2}\left(t_{i}\right)\right)
\end{array}\right], \\
& C_{1}=C_{2}=[1,1], D_{11}=D_{21}=0.1, D_{12}=D_{12}=0.94 .
\end{aligned}
$$


Let $\alpha_{j}=0.5, \varepsilon=0.02$, and obtaining $g_{j}=1, \rho_{j}=0.32$, we get the optimal $H_{\infty}$ performance as the convex optimization problem

$$
\begin{gathered}
\max \gamma \\
\text { s.t. }(15) .
\end{gathered}
$$

Then the optimal $H_{\infty}$ performance gain $\gamma=1.3154$, and the gain matrices of the fault detection filters are

$$
\begin{aligned}
& A_{f 1}=\left[\begin{array}{ll}
-2.0072 & -0.0125 \\
-4.0504 & -1.8172
\end{array}\right], B_{f 1}=\left[\begin{array}{c}
-1.0111 \\
-2.4408
\end{array}\right], \\
& C_{f 1}=\left[\begin{array}{ll}
-0.0045 & 0.1925
\end{array}\right], \quad D_{f 1}=\left[\begin{array}{c}
0.0914
\end{array}\right], \\
& A_{f 2}=\left[\begin{array}{cc}
-1.0119 & 0.2598 \\
0.0664 & -1.8962
\end{array}\right], B_{f 2}=\left[\begin{array}{c}
0.5159 \\
-1.3676
\end{array}\right], \\
& C_{f 2}=\left[\begin{array}{ll}
0.0677 & 0.2011
\end{array}\right], \quad D_{f 2}=\left[\begin{array}{c}
0.4917
\end{array}\right] .
\end{aligned}
$$

At the same time, we also obtain the matrix in Lyapunov function, then the dwell time for each subsystem is obtained with $\delta=1$,

$\left\{\begin{array}{l}\text { dwell time for subsystem } 1 \Rightarrow \tau_{1}>\frac{\ln \left(\left.\mu_{12}\right|_{k_{1}}\right)}{\alpha_{1}}=17.7486 \\ \text { dwell time for subsystem } 2 \Rightarrow \tau_{2}>\frac{\ln \left(\left.\mu_{21}\right|_{k_{2}}\right)}{\alpha_{2}}=11.8165\end{array}\right.$

where $k_{1}, k_{2} \in\{1,2, \cdots\}, k_{1} \neq k_{2}$.

To illustrate the simulation results of the FD objective, the two cases which include the fault for subsystem 1 and subsystem 2, respectively, are considered. The disturbance is assumed to be $d(t)=0.5 \sin (0.5 t) \exp (-0.01 t)$. The switching signal used in this paper in shown in Fig. 2.

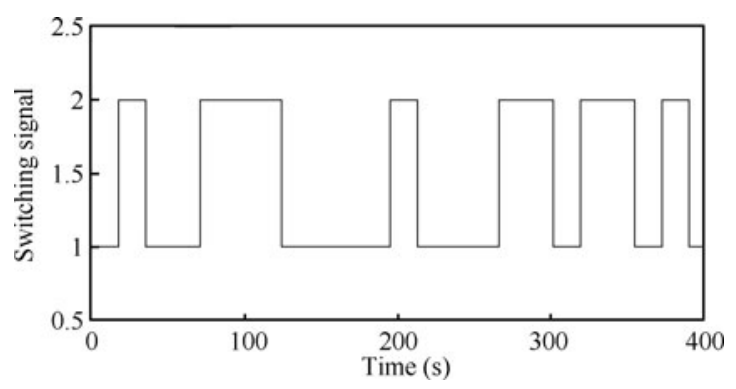

Fig. 2 Switching signal with dwell time $\tau_{1}=17.7486$, and $\tau_{2}=11.8165$

Case 1. The fault for subsystem 1 with the unit amplitude occurs from $100 \mathrm{~s}$ to $200 \mathrm{~s}$. The generated residual $r(t)$ and the evolution of the residual evaluation function $J_{r(t)}$ are shown in Figs. 3 and 4, respectively. It can be seen that when subsystem 1 is activated at $124 \mathrm{~s}$, the residual signal is changed sharply and $J_{r(t)}>J_{t h}$ at $129 \mathrm{~s}$. Thus, the fault for subsystem 1 can be detected.

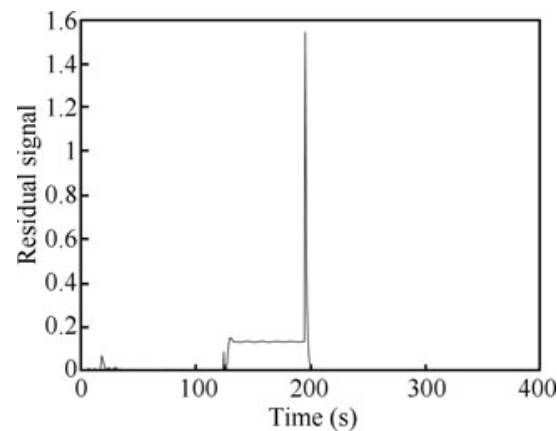

Fig. 3 The residual signal

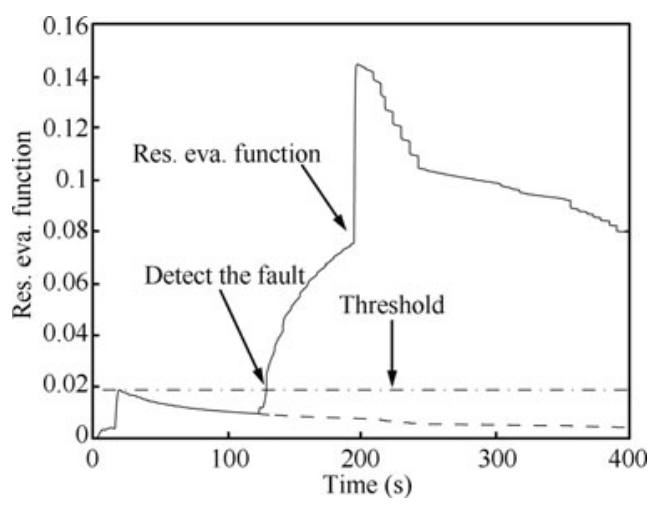

Fig. 4 The residual evaluation function (res. eva. function) and threshold when the fault for subsystem 1 occurs from $100 \mathrm{~s}$ to $200 \mathrm{~s}$

Case 2. The fault for subsystem 2 with the unit amplitude also occurs from $250 \mathrm{~s}$ to $350 \mathrm{~s}$. The generated residual $r(t)$ and the evolution of the residual evaluation function $J_{r(t)}$ are shown in Figs. 5 and 6 , respectively. The simulation results show that when the fault for subsystem 2 occurs, the residual signal varies sharply, and $J_{r(t)}>J_{t h}$ at $266 \mathrm{~s}$, which means that the fault for subsystem 2 can be detected $16 \mathrm{~s}$ after the fault of subsystem 1 occurs. Hence, the fault for subsystem 2 can be detected.

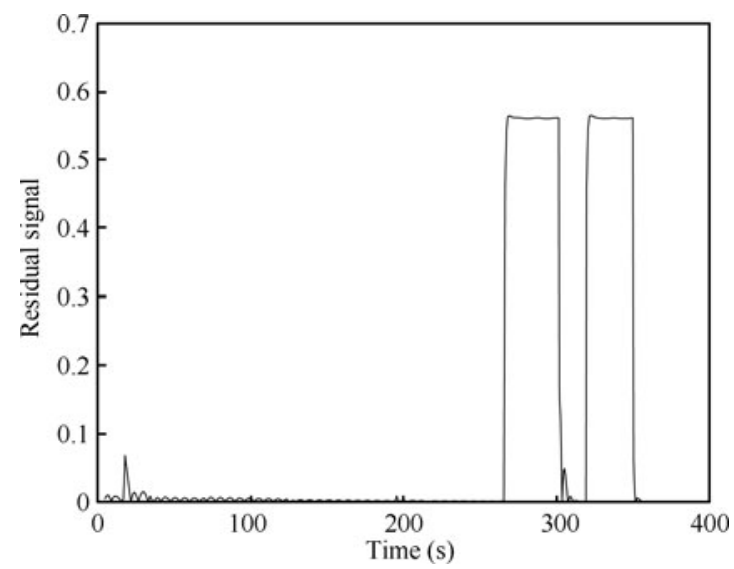

Fig. 5 The residual signal

From Cases 1 and 2, we see that both the fault for subsystem 1 and subsystem 2, respectively, can be detected, and it can demonstrate the effectiveness of the proposed design method. 


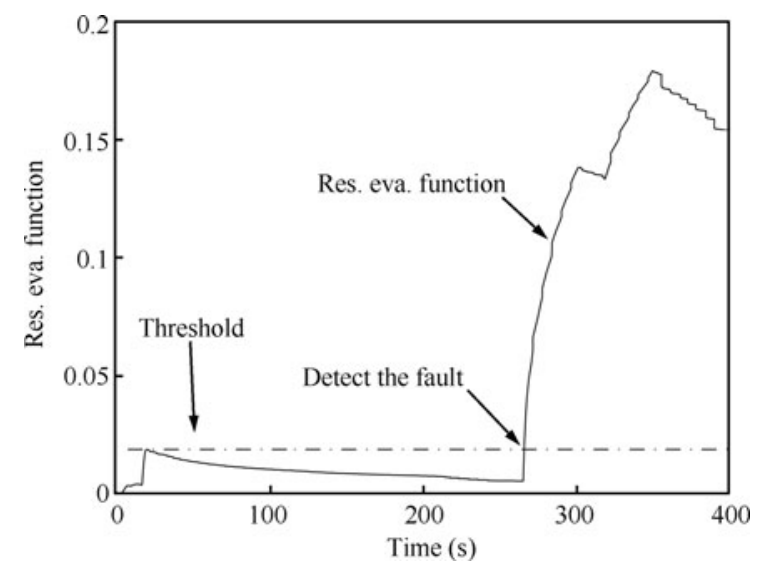

Fig. 6 The residual evaluation function and threshold when the fault for subsystem 2 occurs from $200 \mathrm{~s}$ to $300 \mathrm{~s}$

\section{Conclusions}

This paper investigated the problem of fault detection for a class of nonlinear impulsive switched systems. The $H_{\infty}$ performance to reflect the effect on the residual error signal from disturbances and faults is proposed. In addition, the sufficient condition for the solvability of the FD filters is established by LMIs. Finally, the filters gains are characterized in terms of the solution of a convex optimization problem.

\section{References}

[1] I. Hwang, S. Kim, Y. Kim, C. E. Seah. A survey of fault detection, isolation, and reconfiguration methods. IEEE Transactions on Control Systems Technology, vol. 18, no. 3, pp. 636-653, 2010.

[2] M. Z. Chen, Q. Zhao, D. H. Zhou. A robust fault detection approach for nonlinear systems. International Journal of Automation and Computing, vol. 3, no. 1, pp. 23-28, 2006.

[3] Y. Long, G. H. Yang. Fault detection for a class of nonhomogeneous Markov jump systems based on delta operator approach. Proceedings of the Institution of Mechanical Engineers, Part I - Journal of Systems and Control Engineering, vol. 227, no. 1, pp. 129-141, 2013.

[4] H. Wang, G. H. Yang. A finite frequency domain approach to fault detection for linear discrete-time systems. International Journal of Control, vol.81, no.7, pp.1162-1171, 2008.

[5] X. J. Li, G. H. Yang. Fault detection filter design for stochastic time-delay systems with sensor faults. International Journal of Systems Science, vol. 43, no. 8, pp. 15041518,2012

[6] D. Liberzon. Switching in Systems and Control, Boston, USA: Birkhäuser, 2003.

[7] X. Q. Zhang, J. Zhao. $L_{2}$-gain analysis and anti-windup design of discrete-time switched systems with actuator saturation. International Journal of Automation and Computing, vol. 9, no. 4, pp. 369-377, 2012.
[8] J. Lian, M. Wang. Sliding-mode control of switched delay systems with nonlinear perturbations: Average dwell time approach. Nonlinear Dynamics, vol.62, no. 4, pp. 791-798, 2010.

[9] W. Bao, B. Li, J. T. Chang, W. Y. Niu, D. R. Yu. Switching control of thrust regulation and inlet buzz protection for ducted rocket. Acta Astronautica, vol. 67, no. 7-8, pp. 764$773,2010$.

[10] H. Lin, P. J. Antsaklis. Stability and persistent disturbance attenuation properties for a class of networked control systems: Switched system approach. International Journal of Control, vol. 78, no. 18, pp. 1447-1458, 2005.

[11] L. Zhang, X. W. Gao. Synthesizing scheduled robust model predictive control with target tracking. International Journal of Automation and Computing, vol.9, no. 4, pp.337$341,2012$.

[12] R. C. Ma, J. Zhao. Backstepping design for global stabilization of switched nonlinear systems in lower triangular form under arbitrary switchings. Automatica, vol. 46 no. 11, pp. 1819-1823, 2010.

[13] L. J. Long, J. Zhao. $H_{\infty}$ control of switched nonlinear systems in $p$-normal form using multiple Lyapunov functions. IEEE Transactions on Automatic Control, vol.57, no. 5, pp. 1285-1291, 2012.

[14] D. S. Du, B. Jiang, P. Shi, S. S. Zhou. $H_{\infty}$ filtering of discrete-time switched systems with state delays via switched Lyapunov function approach. IEEE Transactions on Automatic Control, vol. 52, no. 8, pp. 1520-1525, 2007.

[15] S. W. Zhao, J. T. Sun. A geometric approach for reachability and observability of linear switched impulsive systems. Nonlinear Analysis: Theory Methods \& Applications, vol. 72 , no. 11, pp. 4221-4229, 2010.

[16] H. L. Xu, K. L. Teo, X. Z. Liu. Robust stability analysis of guaranteed cost control for impulsive switched systems. IEEE Transactions on Systems, Man, and Cybernetics, Part B: Cybernetics, vol. 38, no. 5, pp. 1419-1422, 2008.

[17] J. Guo, C. Liu, Z. R. Xiang. Robust finite-time $H_{\infty}$ control for impulsive switched nonlinear systems with state delay. Mathematical Problems in Engineering, Article ID 830154, 25 pages, 2012. (Online first).

[18] H. Lin, P. J. Antsaklis. Stability and stabilizability of switched linear systems: A survey of recent results. IEEE Transactions on Automatic Control, vol. 54, no. 2, pp. 308322, 2009.

[19] D. S. Du, B. Jiang, P. Shi. Fault detection for discrete-time switched systems with intermittent measurements. International Journal of Control, vol. 85, no. 1, pp. 78-87, 2012.

[20] D. Wang, P. Shi, W. Wang. Robust fault detection for continuous-time switched delay systems: An linear matrix inequality approach. IET Control Theory \& Applications, vol. 4, no. 1, pp. 100-108, 2010.

[21] J. Li, Q. Y. Su, L. F. Sun, B. Li. Fault detection for nonlinear impulsive switched systems. Mathematical Problems in Engineering, vol. 2013, Article ID 815329, 12 pages, 2013. 
[22] P. M. Frank, X. Ding. Survey of robust residual generation and evaluation methods in observer-based fault detection systems. Journal of Process Control, vol. 7, no. 6, pp. 403424, 1997.

[23] I. R. Petersen. A stabilization algorithm for a class of uncertain linear systems. Systems \& Control Letters, vol. 8, no. 4, pp. 351-357, 1987.

[24] H. L. Xu, K. L. Teo. Exponential stability with $L_{2}$ Gain condition of nonlinear impulsive switched systems. IEEE Transactions on Automatic Control, vol.55, no. 10, pp. 2429-2433, 2010.

[25] L. X. Zhang, N. G. Cui, M. Liu, Y. Zhao. Asynchronous filtering of discrete-time switched linear systems with average dwell time. IEEE Transactions on Circuits and Systems I: Regular Papers, vol. 58, no. 5, pp. 1109-1118, 2011.

[26] D. Corona, J. Buisson, B. De Schutter, A. Giua. Stabilization of switched affine systems: An application to the buckboost converter. In Proceedings of the American Control Conference, IEEE, New York, USA, pp. 6037-6042, 2007.

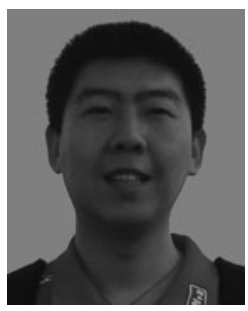

Qing-Yu Su received his B.Sc. degree in electrical automation in 2005, and the M. Sc. degree in control theory and application in 2008, both from Liaoning Technical University, China. He completed his Ph. D. degree in control theory and application in 2013 at Northeastern University, China. Now he is an associate professor at the School of Automation Engineering, Northeast Dianli University, China. He has published 6 articles on SCI Journals and 4 papers on international conferences.

His research interests include switched systems and nonlinear control systems.
E-mail: suqingyuphd@yeah.net (Corresponding author)

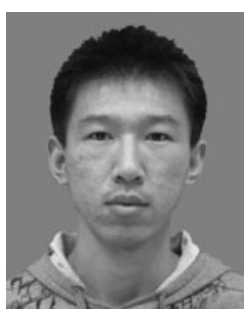

Yan-Cheng Li received his B. Sc. degree in automation in 2013 from Jilin Institute of Chemical Technology, China. Now he is a master student at the School of Automation Engineering, Northeast Dianli University, China.

His research interests include nonlinear control and power systems.

E-mail: liyancheng2012@163.com

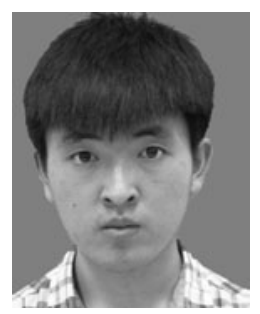

Xing-Ze Dai received his B.Sc. degree in intelligent architecture electrical technology from Zhejiang University of Science and Technology, China in 2013. Now he is a master student at the School of Automation Engineering, Northeast Dianli University, China.

His research interests include micro-grid fault and multi-agent .

E-mail: dazzle1992@126.com

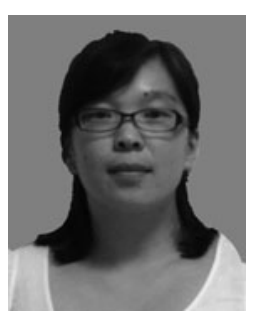

Jian Li received her B.Sc. and M.Sc. degrees in electrical automation and control theory and application from the Liaoning Technical University, China in 2005 and 2008, respectively, and the $\mathrm{Ph}$. D. degree in control theory and application from Northeast University, China in 2013. Currently, she is an associate professor in the School of Automation Engineering, Northeast Dianli University, China.

Her research interests include fault detection, robust control and micro-grid.

E-mail: lijianphd@yeah.net 